\title{
LÍNEAS DE TRABAJO
}

EVALUAR Y EVALUARNOS...

\section{A. Ivancich, D. Petrucci y R. C.Mer- cader}

Taller de Enseñanza de Física, Departamento de Física, Facultad de Ciencias Exactas, Universidad Nacional de La Plata, Argentina.

Un problema esencial en la modificación de las actitudes pasivas de los alumnos que dificulta, y en algunos casos impide, la creatividad y la incorporación de conceptos por parte de ellos, es la evaluación. En un grupode enseñanza-aprendizaje maduro, la evaluación debe servir de parámetro tanto para la institución como para los docentes, pero esencialmente para los propios alumnos.

Aun cuando se logre un clima de mutua confianza en un determinado grupo de alumnos y docentes, la pauta sobre el desarrollo del proceso de enseñanzaaprendizaje debe estar dada por la evaluación. La misma, comprendida en un sentido amplio, no restringido a los exámenes parciales y finales, constituye la instancia en la cual docentes y alumnos constatan si los alumnos han incorporado los contenidos del curso, si la metodología de trabajo en el aula es apropiada para las características del grupo y si los docentes cumplen su rol de facilitadores del aprendizaje. Es indispensable, entonces, abordar los múltiples aspectos de la evaIuación asf́ como sus más variados objetivos; dicho replanteo se dificulta si es unilateral del docente y lleva a situaciones de tensión en los alumnos (Fuertes 1990).

Desde un grupo de docentes que nos proponemos presentar una alternativa en enseñanza, que apunta principalmente a afianzar la función del docente como facilitador del aprendizaje, modificar el rol pasivo del alumno, fomentar la autoestima y el protagonismo en el aula, formar interdisciplinariamente a los alumnos e incentivar la incorporación del método científico, hemos planteado como actividad dentro del aula la discusión del método de evaluación.

Dicha tarea se ha venido planteando sistemáticamente en los cursos de los años 1988,1989 y 1990 . La actividad generadora propuesta en los tres casos, desde principios del año fue la siguiente: a partir de un torbeilino de ideas (brains- torming) sobre la palabra "evaluación», cada alumno eligió una palabra (la que le fuera más significativa) y se formaron grupos de no más de cinco personas, por afinidad de palabra elegida. Estos gru* pos debían trabajar sobre cuatro preguntas (¿por qué evaluar?, ¿qué evaiuar?, ¿quién evalúa? y ¿cómo evaluar?), llegando a una conclusión por grupo, en la cual se debía incluir una propuesta concreta de cómo llevar a cabo una evaluación. Consideramos que dichas preguntas cuestio. nan los aspectos basicos de la evaluación. ya que apuntun tanto al propósito como a fa forma de evaluar. Para cerrar la actividad, se realizó una puesta en común de las conclusiones y a través de un trabajo coordinado, se llegó a una propuesta única de todo el grupo, incluyendo la opinión de los docentes que aportaron su experiencia previa.

Si bien hubo diferencias en los tres años, el elemento común que se encontro es el compromiso de todo el grupo, docentes y alumnos, para encontrar criterios y for. mas de evaluación satisfactorias a todas las expectativas y necesidades; a su vez debía ser cohtrente con el trabajo realizado en las clases.

Las propuestas surgidas y el proceso detaliado de las experiencias en cada año se encuentran en otra publicación (Ivancich 1990). Cabe destacar que en el curso 1988 los alumnos elaboraron problemas para la evaluación parcial, cuya resolu* ción fue grupal y acompañada de un coloquio individual con el docente. Durante el curso de 1989, los alumnos realizaron trabajos de investigacion en grupos (monografías), con exposición grupal y acompañadas rambién por un coloquio individual. Dichos trabajos, profundizados durante todo el curso, se presentaron como evaluación final. Por último, los alumnos del curso 1990 , trabajaron de manera similar al año 1989 , con la diferencia de que los coloquios con los docentes fueron grupales, to cual ayudo a profundizar la autoevaluación de cada alumno.

A partir de los resultados antes mencionados, observamos que, a través de la dinámica grupal en las clases y trabajando sobre el tema evaluación, el grupo logro fijar criterios comunes entre docentes y alumnos, y entre alumnos entre sí; esto implicó un compromiso real con la materia, que permitió un amplio manejo de Ios contenidos $y^{\prime}$ un sinceramiento evidenciado en la autoevaluación de los alumnos y docentes. El porcentaje de alumnos aprobados en cada año fue alto. Es importante destacar que en aquellos casos donde los alumnos no habían a!canzado el grado de conocimiento básico del curso, la decisión de repetir el curso fue tomada de común acuerdo en. tre docentes y alumnos durante el coloquio; en la mayoría de los casos, sugerida por los alumnos. Sólo en un caso, dicho acuerdo no se logró.

Estos resultados iniciales, llevan al grupo docente a considerar la evaluación como un aspecto muy fundamental en pedagogía, digno de incluirse en un proyecto de investigación en enseñanza, en el cual estamos trabajando.

\section{Referencias bibliograficas}

Fuertes, J.F., 1990. Sobre exámenes, Enseñanza de las Ciencias, 8 (2), p. 199.

Wierstra, R.F.A., 1983. Cognitive and affective curriculum-evaluation in the PLON project, Proceeding of the First Intemational Workshop: Research on Physics Education, p. 383.

Ivancich, A., Petrucci D. y Mercader R. C., 1990. Contactos (trabajo enviado para su publicación).

ENSEN̈ANZA-APRENDIZAJE DE LOS CONCEPTOS: SUSTANCIA PURA Y MEZCLA

Estopà Miró, C., Llitjós Viza, A. Departament de Didactica de les Cien. cies Experimentals. Universitat de Bar. celona.

\section{Introducción}

En la escuela no se parte de la nada sino que existe un bagaje de conceptos acumulados por el alumno. Se trata de conseguir, con una metodología científica, cambiar las desviaciones conceptuales en los alumnos. Además, los cursos deberían reflejar mejor la propia actividad científica, de manera que los estudian- 
tes, como futuros ciudadanos o futuros científicos, lleguen a ser más conscientes de las posibilidades y limitaciones de la ciencia como una empresa humana (Driver I988).

Dada la importancia del tema en estudio, que se incluye siempre en los temarios oficiales de ciencias naturales de la enseñanza general básica, se hace imprescindible su conocimiento adecuado por parte de los futuros maestros. El interés del estudio del tema de mezclas y sustancias puras, también se hace evidente por relacionarse con otros temas básicos que son motivo de diferentes estudios: mezclacompuesto químico (Caamaño 1982), fusión-disolución, fusión-combustión (Driver 1985), solución sólido-líquido (Fernández 1988).

\section{Objetivos}

Los objetivos fundamentales del presente trabajo son:

En una primera fase detectar el grado de asimilación de los conceptos de mezcla y sustancia pura en los estudiantes de $\mathrm{Ma}$ gisterio, COU y BUP.

En las fases siguientes observar la evolución de la asimilación de dichos conceptos y el grado de persistencia de las desviaciones conceptuales, después de iratar el tema en clase con una metodología adecuada.

\section{PIanteamiento}

Para la recogida de datos se preparó una encuesta que consta de cuarenta preguntas todas ellas cerradas, a cada pregunta se le asignan tres respuestas. Y se consideró una cuarta posibilidad correspondiente a «no lo sé» para evitar la obligatoriedad de elegir forzosamente una respuesta y aumentar la fíabilidad de los resultados.

La encuesta se aplicó en el curso 19891990.

\section{Resultados}

Los resultados abtenidos se han resumido en la serie de 6 gráficos.

A continuación se presenta uno de ellos como ejemplo.

Tercer curso. Especialidad de Ciencias. Escuela de Magisterio: E-EU

De la interpretación de la gráfica se destacan como conceptos no asumidos:

* La conservación de las propiedades de los componentes de una mezcla y los de transformación física y química (item 1 y 3).

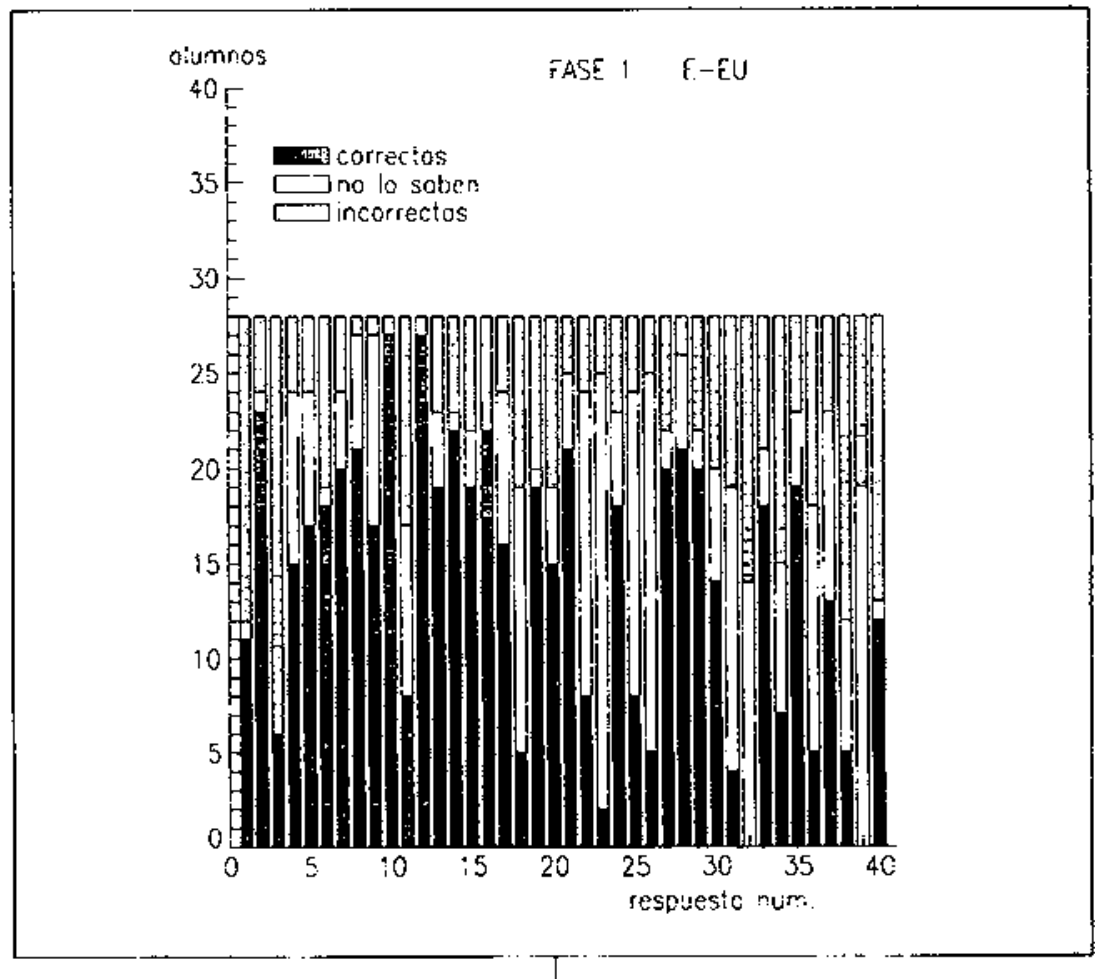

* La palaña fase, para explicar la constitución de una mezcla (ítem 1I)

* La relación entre temperatura de solidificación y temperatura de fusión, y entre temperatura de ebullición y presión de vapor (ftems 36 y 38 ).

* Algunos ejemplos concretos de clasificación de mezclas como el de una solución de sulfato de cobre o incluso el aire (ítems 32,37 y 40 ).

\section{Conclusiones}

Las conclusiones que pueden derivarse del análisis cualitativo de las respuestas al cuestionario no pueden considerarse definitivas, dado que se trata de un estudio preliminar.

Cabe señalar una mayor decisión o una excesiva superficialidad de los alumnos de BUP, cue omitieron casi mayoritariamente la respuesta «no lo sé», lo cual se traduce en un ligero aumento en el número de conceptos no asumidos.

En las respuestas referidas a tos conceptos solución-mezcla homogénea fítem 31) se observa una incoherencia con los resultados de los ítems 6 y 8 referidos a los mismos conceptos. En este caso la utilizacía de un ejemplo concreto produce «desconcierto» en el alumnado.

Curiosamente la mayoría de encuestados, inclutodos tos futuros maestros, no identifican el aire como una mezcla (ftem 40).
De los resultados en los alumnos de magisterio se deriva la necesidad del estudio de disciplinas de contenidos científicos en las escuelas de formación del profesorado. Por este motivo la reforma de los planes de estudio debe contemplar disciplinas de didacticas específicas conjuntamente con las correspondientes a contenidos científicos, por ejemplo: química y su didáctica.

\section{Referencias bibliogrăficas}

Caamaño, A., Mayos, C., Maestre, G. y Ventura, T.,1983. Consideraciones sobre algunos errores conceptuales en el aprendizaje de la química en el bachillerato, Enseñanza de las Ciencias, 1(3), pp. 198-200.

Driver, R., 1985. Children's Ideas In Science. Open University Press. England, pp. 145-169.

Driver, R., 1988. Un enfoque constructivista para el desarrollow currículo en ciencias, Enseñanzakte las Ciencias 6(2); pp. 109-120.

Fernández, J.M., Trigueros, T. y Gordo, L., 1988. Ideas sobre los cambios de estado de agregación y las disoluciones en alumnos de $2^{2}$ curso del BUP, Enseñanza de las Ciencias, 6(1), pp. 42-46. 